\title{
Optimal Harvesting of a Spatial Renewable Resource
}

\author{
Stefan Behringer*
}

\author{
Thorsten Upmann
}

January 15, 2015

\begin{abstract}
In this paper we investigate the optimal harvesting of a renewable natural resource. While in most standard approaches the resource is located at a single point, we allow the resource to be distributed spatially. Consequently, an agent who exploits the resource has to travel from one location to another. For a fixed planning horizon, we investigate the speed and the path of harvesting chosen by the agent. We show that the agent adjusts this speed so as to visit each location only once, even in the absence of travelling cost. Since the agent does not return to any location for a second harvest, it is optimal to fully deplete the resource upon arrival. A similar type of bang-bang solution results when we drop the assumption of a constant harvesting rate: allowing for a variable harvesting rate, the agent chooses to fully exploit the resource either in the last or in the first travelling period. A society interested in conserving some of the resource thus has to take measures to limit the exploitative behaviour of the agent.
\end{abstract}

Keywords: Optimal harvesting; spatial renewable resource; continuous time; market failure; bang-bang solution

JEL classification: Q20, Q28, D21, C61

Acknowledgements: We would like to thank Ginonate Castaldi and participants at LAGV \#11 Marseille; Peter Kort, Nir Becker and participants at the LASER workshop, Brescia; participants of the seminars at IIASA, Laxenburg and Bielefeld University; Lev Lokutsievskiy and participants of the International Conference on Mathematical Control Theory and Mechanics, Suzdal; the participants of the SIRE Workshop on "Finance and Commodities" in St. Andrews; the participants of the NRU HSE workshop in St. Petersburg; and the participants of the "Renewable Resources, Sustainability, and Search" workshop in Heidelberg for valuable comments and discussion. Also, we are grateful to two anonymous referees for helpful comments and suggestions. Finally, the financial support of the German Research Foundation (DFG) is gratefully acknowledged (project no. UP 73/5-1).

\footnotetext{
${ }^{*}$ University of Duisburg-Essen, Mercator School of Management, Lotharstraße 65, 47057 Duisburg, Germany. Email: Stefan.Behringer@uni-duisburg-essen.de

${ }^{\dagger}$ Bielefeld University, Faculty of Business Administration and Economics, Universitätsstraße 25, 33615 Bielefeld, Germany. CESifo, München, Germany. Email: TUpmann@wiwi.unibielefeld.de
} 


\section{Introduction}

The economics of the optimal harvesting of renewable resources is well established. The fundamental papers for the case of fisheries by both Gordon (1954) and Scott (1955) are both almost 60 years old by now. Whereas the former pointed to the problem of overexploitation due to the absence of property rights at sea, the latter established the path of sophisticated dynamic modelling of optimal resource management, providing the foundation for many refined research efforts in more recent decades.

Indicating current research trends and opportunities in natural resource economics, Deacon et al. (1998, p. 390) are critical of the plethora of such refinements due to their tendency to suppress important (but technically challenging) details when seeking analytical insights from simpler constructs. As the most important insights from standard models have already been obtained, an extension of these models should be attempted to incorporate more of the "real world circumstances" with which the managers of fisheries, biologists, and others are concerned.

A most urgent extension of this kind is the recognition of the spatial dimension prevalent in harvesting contexts. Despite its obvious relevance, none of the previous extensions along this line can be found in recent comprehensive textbooks on the topic (e.g. see Conrad, 2010; Perman et al., 2011). Emphasizing this extension, Hannesson (2011a) observes that: "The spatial distribution of fish is rarely analysed in the existing literature, but it could make a difference."

Our aim in this paper is to elaborate on this difference. We thus follow the agenda put forward in Deacon et al. (1998) who also forcefully demand increased realism of resource economics models by acknowledging spatial dimensions: "The spatial dimension of resource use may turn out to be as important as the exhaustively studied temporal dimension in many contexts. Curiously, the profession is only now beginning to move in this direction" (p. 393). In their survey paper on "The economics of spatial-dynamic processes," Smith, Sanchirico, and Wilen (2009) similarly note that whereas there is a long tradition in resource economics of being concerned with the dynamic aspects of resource use, and economics has a long history addressing spatial aspects of economic activity, the two approaches have rarely been integrated into a single model ever since Hotelling separated them in his two seminal papers (1929) and (1931).

Some authors have taken this call seriously. Recent work that simultaneously allows for spatial characteristics and a time dimension includes Sanchirico and Wilen (2005) and Costello and Polasky (2008). Both papers work within the framework of meta-population models with discrete patches, but with connectivity 
between these (e.g. diffusion). ${ }^{1}$ Sanchirico and Wilen (2005) characterize the optimal way to distribute harvesting effort over space and time in order to maximize discounted profit and compare it with the results derived when ignoring these spatial processes. Once the biological dispersal process is further specified, optimal instruments are shown to be sensitive to spatial gradients of both rents and the chosen disposal, and first and second best solutions are compared.

Costello and Polasky (2008) allow for a meta-population model with random events, that is, stochastic growth of the resource within each patch and stochastic dispersal of the resource between patches. Economic variables can also be spatially heterogeneous. In this very general setting they are able to derive optimal spatially explicit harvesting strategies that maximize the expected present value of profit from harvesting. Whereas interior solutions will be time and state independent, the optimal strategy will in general vary across space. In the case of corner solutions, it may be optimal to close some patches for some periods.

Similar to Deacon et al. (1998), Smith, Sanchirico, and Wilen (2009, p. 105) conclude that "research addressing integrated spatial-dynamic processes is needed and arguably overdue." Wilen (2007, p. 1135) contrasts this lack of attention by (resource) economists with the prominence of spatial dynamic systems in the hard sciences such as mathematics and physics whose tools have been employed by scholars even to study biological and ecological issues (see e.g. Neubert, 2003; Kellner et al., 2007; and Neubert and Herrera, 2008). The latter assume that the fish itself can move by introducing a diffusion coefficient. This particular extension, where the resource is assumed to move from areas of high to low concentration, is also taken up in recent comprehensive work of Brock and Xepapadeas (2010) and their investigation of commercial fishing in Brock, Xepapadeas, and Yannacopoulos (2013), and allows them to investigate robust methods to control such interconnected spatiotemporal systems.

An alternative approach is to bundle the choice of an agent's harvesting speed with the amount that can be extracted, making the analysis more manageable. This approach, which is usually referred to as a search model, has been followed by a series of papers, among them Robinson, Williams, and Albers (2002) and Robinson, Albers, and Williams (2008) in a resource extraction (timber gathering) model. A similar approach has been chosen by Belyakov, Davydov, and Veliov (2013), whose paper is the closest to ours. These authors make similar assumptions about the spatial dimension of the renewable resource (that is, it is a single-aged, homogeneous population of a motionless resource $)^{2}$ and the harvesting technology.

\footnotetext{
${ }^{1}$ Further examples of this line of research are given in Smith, Sanchirico, and Wilen (2009, fn 11).

${ }^{2}$ As pointed out by an anonymous referee, this is equivalent to assuming a continuum of independent homogeneous stocks. With an age-homogeneous population, spatial diffusion of the
} 
However, while their model also allows for heterogeneous space, the speed of movement and the harvesting intensity are interdependent, thereby effectively reducing the set of controls to a singleton. Also, the harvesting agent may decide to wait for some time in each round, but will optimally decide not to do so if the heterogeneous data of the model reveals a sufficiently large regeneration-harvesting effectiveness ratio. In our setting, though, the speed and the harvesting intensity are treated as separate choice variables (this was motivated by thinking about fishing nets as harvesting tools), while temporary waiting is not allowed. Also, in Belyakov, Davydov, and Veliov (2013) there is no discounting of future yield. Still, their approach has important similarities in method and intuition and should be seen as highly complementary to our paper.

While we believe our approach to be more general and broadly applicable, e.g. to agriculture and various renewable natural resources, we follow the literature and use the case of fishery for illustration and motivation. ${ }^{3}$ The critical point from which virtually all of the existing resource models abstract is that fish are (as are other resources) distributed spatially, namely in oceans, seas, and rivers. A fisher thus has to travel by boat to catch fish at each spot visited.

We assume that the boat starts at some harbour, follows a given route, ${ }^{4}$ and eventually returns to its point of departure. The time of this journey (round-trip) depends on the speed of the boat, which is controlled by the fisher. We assume that the planning horizon of the fisher is finite. This may be interpreted as either that the fisher is concerned with only one season of fishing (or harvesting), or possesses a fishing license with fixed finite maturity, or that the planning horizon equals the fisher's working lifetime - and other interpretations may also come to mind. For this fixed planning horizon, the number of journeys that can be undertaken also depends on the speed of the boat. As all fishing is done using fishing nets, the fisher has control over the fraction of the stock to be caught by the choice of mesh size, boat type, and effort.

It is a common precept that society has a concern for sustaining wildlife in the oceans and seas. Also, it is well known (as emphasized by Gordon, 1954) that

$\overline{\text { resource would }}$ result in inflows to and outflows from a location to net out. Therefore, only if we allowed for a heterogeneous population and diffusion would movements affect the age-structure of the resource. Since the focus of our paper, similar to Belyakov, Davydov, and Veliov (2013), is on the movement of the agent, not that of the resource, we refrain from adding these additional complications at this point to the benefit of clear cut analytical results.

${ }^{3}$ Since the resource will not move, the reader may prefer to think of the resource as some (generic) plant or agricultural product rather than fish.

${ }^{4}$ The assumption of a route given at the beginning of the trip does not represent any restriction as long as there is no uncertainty about the location of the resource and hence no necessity to search: we may simply think of the given route as the most lucrative route available, determined beforehand. 
there exist critical externalities resulting from the general absence of property rights. In the case of a renewable resource such as fish, sustainability can also be in the interest of the fisher, as a fish stock of a given size that has not been fully harvested will recreate itself after some time, allowing for a larger catch in the future. Yet, for any reasonable modelling of costs, our model reveals that it will be privately optimal for the fisher to fully exploit the fish stock by only making the journey once. Thus, the fisher adjusts the speed of the boat in a way so as to complete exactly one round of travelling, and will therefore deplete the resource totally.

From a technical perspective, our solution can be seen as a robustness check for the solution of non-spatial models with similar assumptions about the data where the solution will also be of the bang-bang type. If we dispense with the assumption of a constant harvesting rate and the agent can vary the rate freely, a form of bang-bang solution prevails. The timing of total exploitation now depends on whether the growth rate of the resource exceeds the discount rate. Whenever it remains optimal for the fisher to go for only one fully exploitative round (and is not allowed to wait), the two solutions - harvesting in the first and harvesting in the last round - coincide. Moreover, we show that this result continues to hold if we allow for travelling costs.

Clearly, with extinction being irreversible, this outcome cannot be in the interest of society at large. As it is very costly to control the actual catch (see Hannesson, 2011b, for details), society has to consider alternative mechanisms and policies to restrict the amount of fish caught. One possible remedy for overexploitation would be to guarantee that the agent undertakes several fishing journeys. With multiple journeys within the fixed time horizon, it will then be in the agent's private interest not to over-exploit the resource in the first visit. The underlying idea is that with multiple journeys, the tendency to over-exploit the resource is mitigated as the remaining stock will recover after some time providing the potential for an even larger catch in the future.

An alternative instrument in fishery management is to grant the fisher a residual or salvage value for the stock of the resource remaining at the end of the fishing period. Rather than imposing an input control on the fishing activity, this policy aims directly at the fisher's economic incentives: with a salvage payment increasing in the remaining stock of the resource, it is in the fisher's own economic interest not to exhaust the stock unduly. When chosen appropriately, both policy instruments are equivalent in terms of conservation of the stock of the resource.

Also, an instrument frequently observed in practice is the establishment of harvesting quotas or catch shares. Here, a regulator determines a species-specific 
total allowable catch, and allocates portions of this catch to individuals, institutions, or countries. While originally designed to prevent over-exploitation of a common property resource by multiple agents, these instruments may be applied even if only a single agent has access to the resource. If social and private interests diverge, society may impose harvesting quotas or catch shares to prevent overfishing irrespective of the number of fishers.

Finally, a complementary instrument, which our model allows for explicitly, is the specification of a minimum mesh size. This policy lets small fish to escape and thus provides the conditions for replenishing the stocks. All of these instruments are intended to preserve the stocks and to promise higher future yields at the cost of a smaller catch today.

The rest of this paper is structured as follows: In Section 2 we describe our formal model. In Section 3 we characterize the agent's optimal harvesting policy when bound to choose a constant speed along with a constant harvesting rate. In Section 4 we demonstrate that our results are robust to possible costs of movement. A detailed analysis of the first period can be found in Section 5. In Section 6 we show that our result of a totally exhaustive harvesting round prevails even if we do not require the agent to choose a constant harvesting rate. We conclude in Section 7.

\section{The Model}

Consider an economic agent (we may think of a fisher), ${ }^{5}$ exploiting or cutting a renewable natural resource (e.g. a fish stock). Since this resource expands over the space, harvesting requires the agent to travel from one location to another. That is, when the yield at one particular point in the space is collected, the agent has to move to the next location in order to proceed with the harvesting. Movement, though, may be costly, but this cost can be avoided by reducing the speed of movement - and instead exploiting the resource at each point more severely. While a lower speed implies foregone revenue from the yield of the subsequent points in space that cannot be reached within the given time frame, an intensified extraction leaves the stock with less beneficial conditions for future growth of the resource. The agent thus has to choose both the speed of the movement and the amount to be harvested at each point within this region. We assume that the harvesting capacity of the agent is fixed so that at each point in time the harvest is bounded from above. In other words, we assume that at any point in time and space the agent may collect any non-negative amount of the resource which neither exceeds the stock nor the fixed harvesting capacity.

\footnotetext{
${ }^{5}$ We henceforth speak of the agent and the fisher interchangeably.
} 
For the sake of tractability, we reduce the dimension of the extension of the resource. Instead of literally modelling the extension of the resource and the movement of the agent in a two-dimensional space, we consider a one-dimensional setting. We assume that the resource is located on the periphery of a unit circle and that the movement of the agent is a journey on this periphery. ${ }^{6}$ We therefore have to keep track of time and location. We denote the fixed time horizon (harvesting period) by $\mathcal{T} \equiv[0, T]$, an instant of time within it by $t \in \mathcal{T}$, a fixed location by $x \in \mathcal{S} \equiv[0,2 \pi]$, and the location of the agent at time $t$ by $s(t) \in \mathcal{S}$. The size of the stock at location $x$ at time $t$ is denoted by $f(t, x)$. Thus $f(t, \cdot)$ is the distribution of the resource on the periphery at time $t$.

The natural resource is autonomously growing at rate $g$. We allow the growth rate of the stock at a particular location to depend on the stock, but neither on time nor on location directly. That is, we assume that the growth function does not change in time and is the same for all locations on the periphery. The growth of the stock is governed by the differential equation $f_{t}(t, x) \equiv \frac{\partial}{\partial t} f(t, x)=$ $g(f(t, x)), \forall x \in \mathcal{S}, t \in \mathcal{T}^{7}{ }^{7}$ except at some finite set of points of discontinuity of $f(\cdot, x)$. In addition, the stock of the resource at location $x$ is reduced by the harvest at time $t$ whenever the agent's location at time $t$ equals $x$, i.e., $s(t)=x$. If we denote the extraction of the resource at time $t$ at point $x$ by $q(t, x)$ and the agent's harvest by $h(t)$, we have $h(t) \equiv q(t, s(t))$ and $q(t, x)=0, \forall x \neq s(t)$. This reflects the fact that extraction at point $x$ may only take place if $x$ is the actual location of the agent - and if so, the harvest leads to a jump of the stock by $\Delta f(t, x) \equiv f\left(t^{+}, x\right)-f\left(t^{-}, x\right)=-h(t)=-q(t, s(t)){ }^{8}$ Thus, for any given location $x$, the set of arrival times of the agent at $x, J(x):=\left\{t_{1}(x), t_{2}(x), \ldots\right\}$, equals the set of times of (potential) jumps in the stock $f(\cdot, x)$. Putting the pieces together, the stock of the resource obeys the law of "motion"

$$
\begin{aligned}
f_{t}(t, x) & =g(f(t, x)) & & \forall t \in \mathcal{T} \backslash J(x), x \in \mathcal{S}, \\
f\left(t^{-}, x\right)-f\left(t^{+}, x\right) & =h(t) & & \forall t \in J(x), x \in \mathcal{S},
\end{aligned}
$$

with $f(0, x)=f_{0 x}$ for all $x \in \mathcal{S}$. Note that although $h$ is non-negative for all $t \in \mathcal{T}$, harvesting only leads to a jump in $f(\cdot, x)$ if and when the agent arrives at location $x$, that is at times $t \in J(x)$.

${ }^{6}$ Clearly, fishing nets are neither dimensionless, nor are fishing grounds one dimensional in the real world. However, our model may accommodate such extensions easily, as any twodimensional fishing route may be projected onto a line (here the periphery of a circle), and any one dimensional fishing net onto a single point (on that line).

${ }^{7}$ We assume $g(y) \geq 0$ on some interval $[\underline{y}, \bar{y}]$.

${ }^{8} t^{+}$denotes the limit on the right at $t: t^{+} \equiv \lim _{s \searrow t} s$; and $t^{-}$, the limit on the left at $t$ : $t^{-} \equiv \lim _{s \nearrow t} s$. 
As the resource is distributed over the periphery of the circle, the agent is required to travel along the periphery in order to proceed with harvesting from one location to the next. There are two natural specifications of how the speed may be controlled: either the agent can control the speed of movement $v$ directly, or cannot control $v$ directly but only the acceleration. We follow the former approach for now. Note that the law of motion of the resource does not depend on the speed of the agent, but only on the local harvesting activity.

At any instant of time, the agent faces a capacity constraint $\bar{h}$ limiting the possible harvest at each location to $[0, \bar{h}]$. At each moment the agent's harvest is therefore restricted to $h(t) \in H(t):=[0, \min \{\bar{h}, f(t, s(t))\}], \forall t \in \mathcal{T}$. The problem of the agent is then to maximize the discounted profit flow consisting of instantaneous revenue net of instantaneous cost $C(v(t), h(t))$, which generically depends on both speed $v$ and harvest $h$, for a given planning horizon $\mathcal{T}$. Let $\rho \geq 0$ denote the discount rate of the agent, and normalize the price of one unit of the harvested resource to unity, ${ }^{9}$ then the optimisation problem is

$$
\begin{aligned}
\max _{\{v, h\}} & \int_{0}^{T} e^{-\rho t}(h(t)-C(v(t), h(t))) d t \\
\text { s.t. } \quad \dot{s}(t) & =v(t), \quad \forall t \in \mathcal{T} \\
f_{t}(t, x) & =g(f(t, x)) \quad \forall t \in \mathcal{T} \backslash J(x), x \in \mathcal{S} \\
f\left(t^{-}, x\right)-f\left(t^{+}, x\right) & =h(t), \quad \forall t \in J(x), x \in \mathcal{S} \\
h(t) & \in H(t), \quad \forall t \in \mathcal{T} \\
f(0, x) & =f_{0 x}, \quad \forall x \in \mathcal{S} \\
s(0) & =0,
\end{aligned}
$$

The last condition requires the agent to start, without loss of generality, at location 0 . In addition, we may also require the agent to terminate the trip at the point of departure, that is, require $s(T)=s(0)=0$. While this represents a reasonable restriction when the agent can only terminate the trip at home, we allow for the fisher to end the trip at any point (that may also be a harbour) along the route. Yet, as we shall see, even though we allow the agent to go for any real-valued number of rounds, the agent decides to travel complete rounds.

\footnotetext{
${ }^{9}$ Our assumption of a revenue that is linear in the harvest $h$ implies classical competitive behaviour of the agent. While we think that price-taking is a reasonable assumption for our motivational example, we also do not want our focus on optimal harvesting with a spatial structure to be obstructed by an analysis of non-competitive behaviour at present.
} 


\section{Constant Speed and Constant Harvesting Rate}

For convenience, we disregard an initial acceleration period and assume that the agent may immediately start with some non-negative speed which is maintained until time $T$. Whence we assume that the agent circulates with some constant speed $v(t)=v$ at all times $t \in \mathcal{T}$. Also, here we disregard the harvesting cost. In this way, the analysis of this section represents a first step towards a thorough solution of Problem (3). In particular, we take into account the harvesting cost in Section 4, provide a detailed analysis of the first period in Section 5, and eventually allow for non-constant harvesting rates in Section 6. In each of these cases we show how and to what extent the results of this section will continue to apply.

3.1. Evolution of the stock at a fixed location. Assume that the growth function $g$ is linear, ${ }^{10}$ i.e., $g(y)=r y$, and that the initial stock is constant on the periphery of the circle, i.e., $f(0, x)=y_{0}$. Taken together, these conditions imply for each $x \in \mathcal{S}=[0,2 \pi]$ :

$$
f_{t}(t, x)=r f(t, x) \quad \text { with } \quad f(0, x)=y_{0}, \quad \forall t \in \mathcal{T} \backslash J(x), x \in \mathcal{S},
$$

yielding

$$
f(t, x)=f(0, x) e^{r t}=y_{0} e^{r t}=: \tilde{f}\left(t, y_{0}\right),
$$

provided that $q(s, x)=0, \forall s \in[0, t]$. Since the agent does harvest, we now derive the evolution of the stock taking into account (interim) harvesting activity.

Assuming the agent travels with constant speed along the periphery, i.e., $v(t)=v, \forall t \in \mathcal{T}$, we define the time necessary to traverse the periphery of the circle once by $\theta(v) \equiv 2 \pi / v$ (see Figure 1 ). Thus $v /(2 \pi)=1 / \theta(v)$ represents the frequency or speed of circling. Simplifying $\theta(v)$ to $\theta$ from now on, we may express this and the subsequent formulae in terms of either circling time $\theta$ or speed $v$.

The time of the first passage of the location $x \in[0,2 \pi]$ then equals $t_{1}(x)=$ $\frac{\theta x}{2 \pi}=\frac{x}{v}$, and the size of the stock at this moment is given by

$$
f\left(t_{1}(x), x\right)=y_{0} \exp \left(r \frac{\theta x}{2 \pi}\right)=y_{0} \exp \left(r \frac{x}{v}\right) .
$$

More generally, we define $t_{n}(x)$ as the time of the $n$th arrival at location $x$, which is given by

$$
t_{n}(x) \equiv(n-1) \theta+\frac{\theta x}{2 \pi}, \quad \forall n \in \mathbb{N} \backslash\{0\} .
$$

\footnotetext{
${ }^{10}$ Clearly, exponential growth cannot prevail for sufficiently large values of the stock due to the limited carrying capacities of the environment. So this growth process is to be understood as an approximation of the evolution of a stock below a critical size - which constitutes the relevant and interesting case.
} 


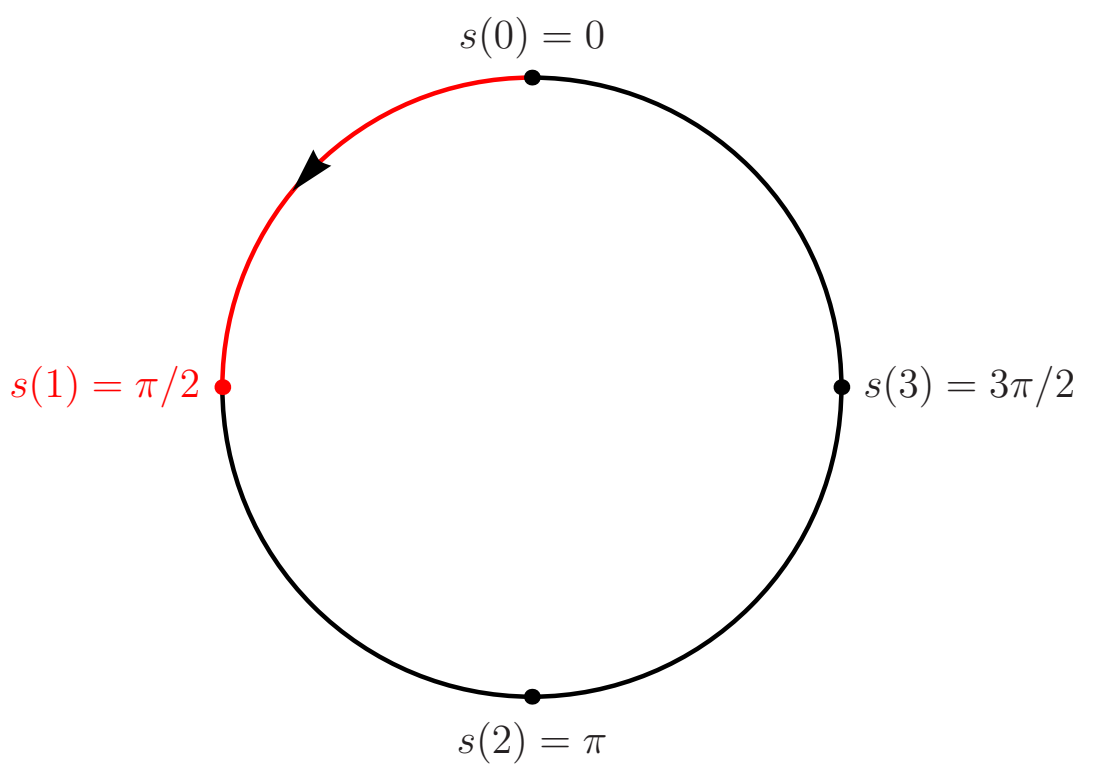

FiguRE 1. Movement of the agent on the periphery with constant speed $\theta=4$.

Without (interim) harvesting, the size of the stock of the resource at the time of the agent's $n$th arrival at location $x$ equals

$$
y_{0} \exp \left((n-1) r \theta+r \frac{\theta x}{2 \pi}\right)=y_{0} \exp \left(\frac{r}{v}(2 \pi(n-1)+x)\right)
$$

which is displayed in Figure 2.

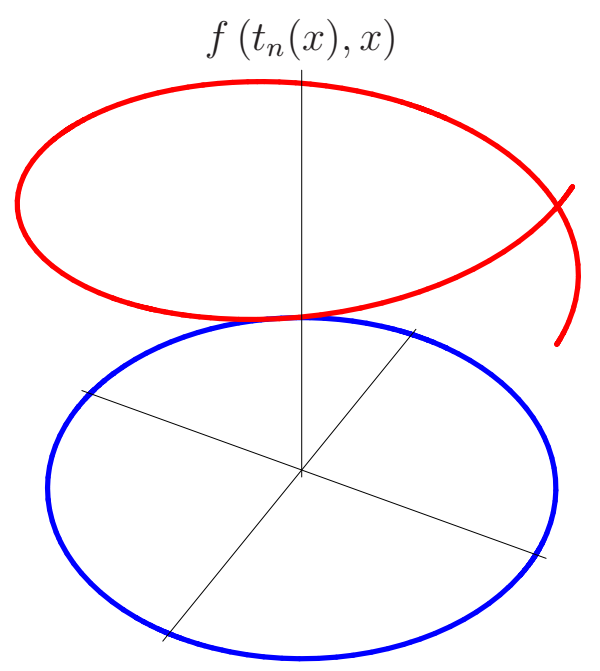

FIGURE 2. Size of the stock of the resource at time of arrival: $f\left(t_{n}(x), x\right)$.

Assuming that at each location $x$ the agent harvests a constant fraction of the stock, say $1-\alpha(\alpha \in[0,1])$, the stock at location $x$ in the $n$th period immediately 
before the $n$th harvest amounts to

$$
f\left(t_{n}(x), x\right)=y_{0} \alpha^{n-1} \exp \left((n-1) r \theta+r \frac{\theta x}{2 \pi}\right),
$$

and accordingly the stock at $x$ after the $n$th time of passing this location equals

$$
y(x, n) \equiv f\left(t_{n}^{+}(x), x\right)=y_{0} \alpha^{n} \exp \left((n-1) r \theta+r \frac{\theta x}{2 \pi}\right), \quad \forall n \in \mathbb{N} \backslash\{0\} .
$$

Define $y(x, 0) \equiv y_{0}$. It is important to realize that $y(x, n)$ equals the starting value of the $n+1$ th growth period of the resource at location $x$. With this initial value of each round of growth at hand, we can now define the effective growth time since the last arrival of the agent at location $x$ :

$$
\tau(x, t)= \begin{cases}t & \text { if } \quad 0 \leq t<\frac{\theta x}{2 \pi} \\ \bmod \left(t-\frac{\theta x}{2 \pi}, \theta\right) & \text { if } t \geq \frac{\theta x}{2 \pi}\end{cases}
$$

where mod denotes the modulo-function. Defining $Q(x, y) \equiv\left\lfloor\frac{x}{y}\right\rfloor$ as the integer quotient of real numbers $x$ and $y, Q(T, \theta)$ denotes the maximal number of complete rounds which can be completed in time $T$ if the time required to complete one round equals $\theta$. Here $\lfloor\cdot\rfloor$ denotes the floor function, yielding the greatest integer less than or equal to its argument. Correspondingly, $\bmod (T, \theta) \equiv T-\theta Q(T, \theta)$ denotes the time remaining after the maximal number of rounds in time $T$ with circulating time $\theta$ has been completed. Consequently the number of times the agent has passed location $x$ at time $t$ is given by

$$
m(x, t)=\left\lfloor\frac{t-\frac{\theta x}{2 \pi}}{\theta}\right\rfloor+1 .
$$

Using $y, \tau$, and $m$ we may now derive the resulting stock of the resource at location $x$ and time $t$ :

$$
f(t, x)=\tilde{f}(\tau(x, t), y(x, m(x, t))) .
$$

The resulting stock is displayed (for $x=\pi / 2, \theta=4, y_{0}=1$ and $\alpha=2 / 3$ ) in Figure 3.

3.2. Aggregate harvest. We now derive the present value of the aggregate harvest. Recall that the discount rate equals $\rho \geq 0$. If we sum over all $x \in[0,2 \pi]$ the discounted harvest at location $x$, we obtain for the first round of circling

$$
\begin{aligned}
E(1) & =(1-\alpha) y_{0} \int_{0}^{2 \pi} \exp \left((r-\rho) t_{1}(x)\right) d x \\
& =(1-\alpha) y_{0} \int_{0}^{2 \pi} \exp \left((r-\rho) \frac{\theta x}{2 \pi}\right) d x \\
& =(1-\alpha) y_{0} \frac{2 \pi}{(r-\rho) \theta}\left(e^{(r-\rho) \theta}-1\right),
\end{aligned}
$$




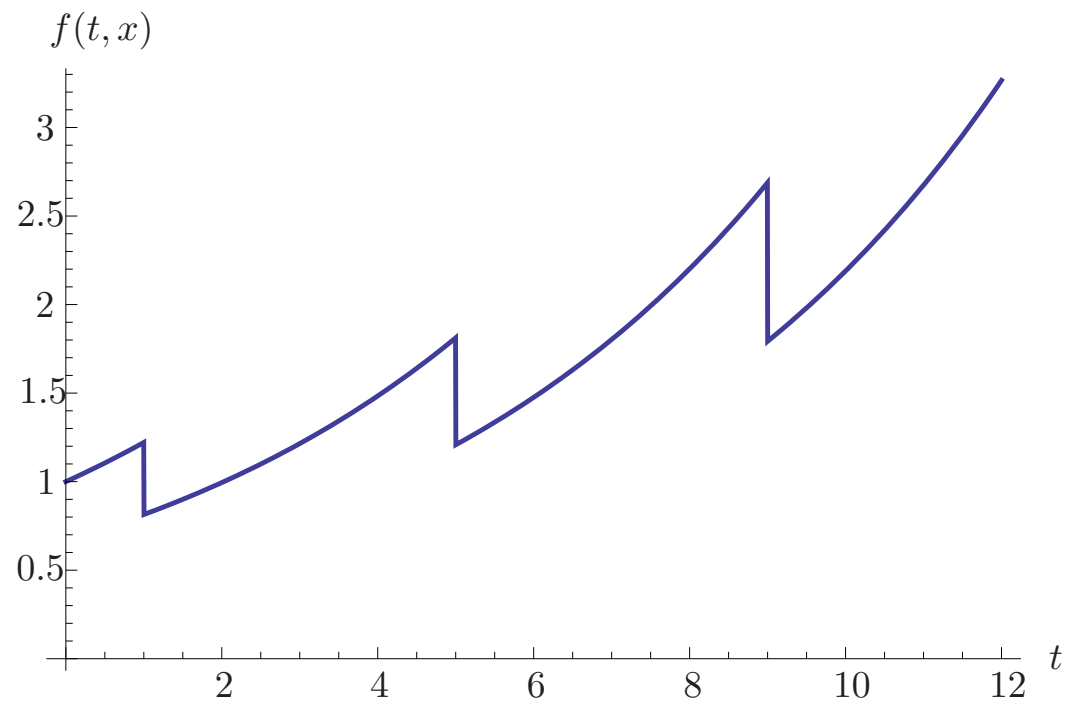

Figure 3. Growth of the stock at location $x=\pi / 2$ (with $\theta=$ $\left.4, y_{0}=1, \alpha=2 / 3\right)$

where we have made use of the fact that $t_{1}(x)=\frac{\theta x}{2 \pi}$ is the time of first passage of location $x$, and that $\theta=2 \pi / v$ is the time necessary to traverse the periphery once.

Similarly, the total discounted harvest of the $n$th period equals

$$
\begin{aligned}
E(n) & \equiv(1-\alpha) \alpha^{n-1} y_{0} \int_{0}^{2 \pi} \exp \left(-\rho t_{n}(x)\right) \exp \left((n-1) r \theta+r \frac{\theta x}{2 \pi}\right) d x \\
& =(1-\alpha) \alpha^{n-1} \frac{2 \pi y_{0}}{(r-\rho) \theta}\left(e^{(r-\rho) \theta}-1\right) e^{(n-1)(r-\rho) \theta}
\end{aligned}
$$

where we have made use of the definition of the general arrival time $t_{n}(x)$ as defined in Equation (4). More generally, let us define $E(n, x)$ as the discounted harvest of the $n$th period up to location $x$, which is given by

$$
\begin{aligned}
E(n, x) & \equiv(1-\alpha) \alpha^{n-1} y_{0} \int_{0}^{x} \exp \left(-\rho t_{n}(\xi)\right) \exp \left((n-1) r \theta+r \frac{\theta \xi}{2 \pi}\right) d \xi \\
& =(1-\alpha) \alpha^{n-1} \frac{2 \pi y_{0}}{(r-\rho) \theta}\left(e^{(r-\rho) \frac{\theta x}{2 \pi}}-1\right) e^{(n-1)(r-\rho) \theta}
\end{aligned}
$$

Now $E(x, n)$ is the present value of the harvest of period $n$ if harvesting is only done for locations in $[0, x]$ but not for those in $(x, 2 \pi]$. 
Finally, summing the per-period harvest over all periods up to period $n$, we obtain the aggregate harvest after $n$ growth and harvesting periods: ${ }^{11}$

$$
\begin{aligned}
\sum_{i=1}^{n} E(i) & =(1-\alpha) y_{0} \frac{2 \pi(\exp ((r-\rho) \theta)-1)}{(r-\rho) \theta} \frac{\left(1-\alpha^{n} \exp (n(r-\rho) \theta)\right)}{1-\alpha \exp ((r-\rho) \theta)} \\
& =(1-\alpha) \frac{2 \pi y_{0}}{(r-\rho) \theta} \frac{\left(e^{(r-\rho) \theta}-1\right)\left(\alpha^{n} e^{n(r-\rho) \theta}-1\right)}{\alpha e^{(r-\rho) \theta}-1}
\end{aligned}
$$

If $s(T) \neq 0$, that is, if the final period ends before the agent completes the last round-trip, we have to add the resulting fraction of the last period. Adding to the above sum the term $E(n+1, s(T))$, we arrive at the discounted aggregate harvest collected within time $T$,

$$
\begin{aligned}
& G(\theta, \alpha) \equiv \sum_{i=1}^{Q(T, \theta)} E(i)+E(Q(T, \theta)+1, s(T)) \\
& =(1-\alpha) \frac{2 \pi y_{0}}{(r-\rho) \theta}\left(\alpha^{Q(T, \theta)}\left(e^{(r-\rho) \bmod (T, \theta)}-1\right) e^{\theta(r-\rho) Q(T, \theta)}\right. \\
& \left.+\frac{\left(e^{\theta(r-\rho)}-1\right)\left(\alpha^{Q(T, \theta)} e^{\theta(r-\rho) Q(T, \theta)}-1\right)}{\alpha e^{(r-\rho) \theta}-1}\right) .
\end{aligned}
$$

For any fixed time horizon $T, G$ represents the discounted gross payoff of the agent travelling the periphery with circling frequency $1 / \theta$ while harvesting the fraction $1-\alpha$ of the stock at each location. From this gross payoff the agent has to subtract the cost of motion.

We now investigate the properties of $G$. For any given location $x$, the stock, more precisely the function $f(\cdot, x)$, is discontinuous at the time of the arrival of the agent. Now, accounting for the movement of the agent and continuity of the growth function, we see that the stock of the resource along the path of the agent $f\left(t_{n}(\cdot), \cdot\right)$ is also continuous, unless the agent completes a full circle, which happens at location $x=0$. Since the harvest is proportional to the stock, $h(t)$ is also discontinuous only at times $t \in J(0)$. This implies that $G(\cdot, \alpha)$ has kinks whenever $\bmod (T, \cdot)=0$ and is thus not differentiable at these points. However between any two adjacent kinks, say $\theta_{1}$ and $\theta_{2}, G(\cdot, \alpha)$ is differentiable and convex as can be seen from Figure 4 . Note that $G(\cdot, \alpha)$ is not necessarily quasi-concave, as the green graph in Figure 4 reveals. On the other hand $G(\theta, \cdot)$ is differentiable and quasi-concave as displayed in Figure 5. (In each figure the graph of the lowest parameter value is displayed in red.)

\footnotetext{
${ }^{11}$ Note that we do not need to assume $r>\rho$, i.e., that the growth rate exceeds the discount rate. In fact, as we show in Appendix $\mathrm{A}, E(n, x)$ is always positive and the optimal solution to our problem does not depend on whether $r-\rho$ is positive or negative.
} 


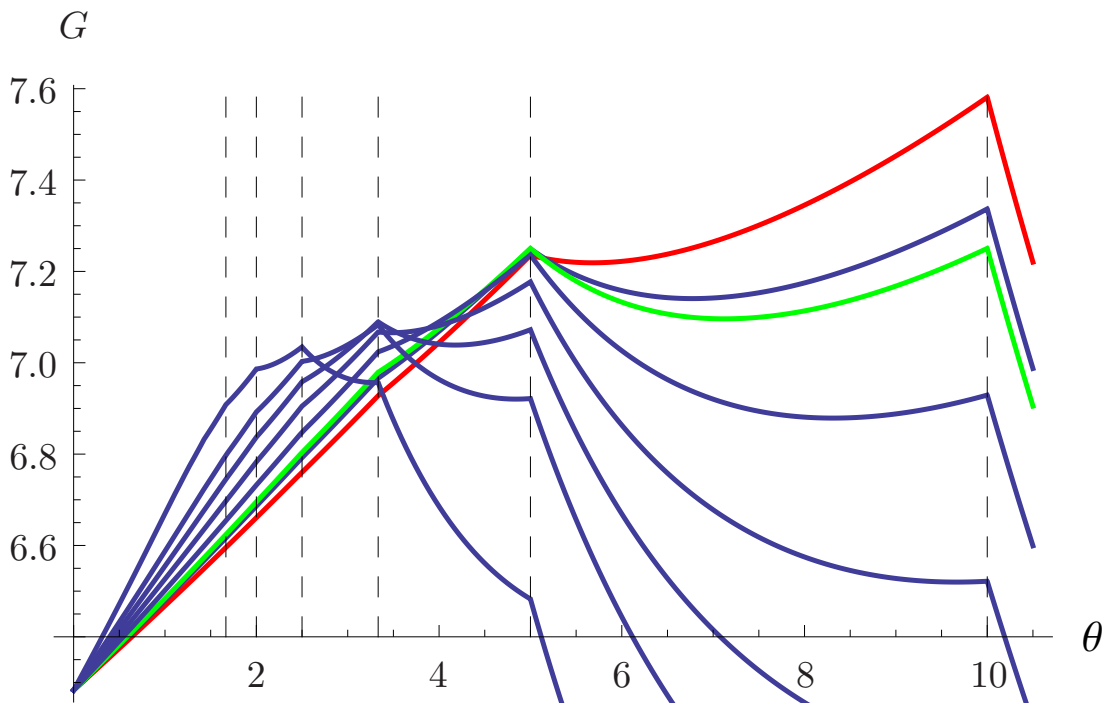

Figure 4. $G$ evaluated at $\alpha=0.07,0.1,0.1106,0.15,0.2,0.25,0.3$, 0.4 (with $\left.y_{0}=1, r=1 / 10, \rho=1 / 20, T=10\right)$.

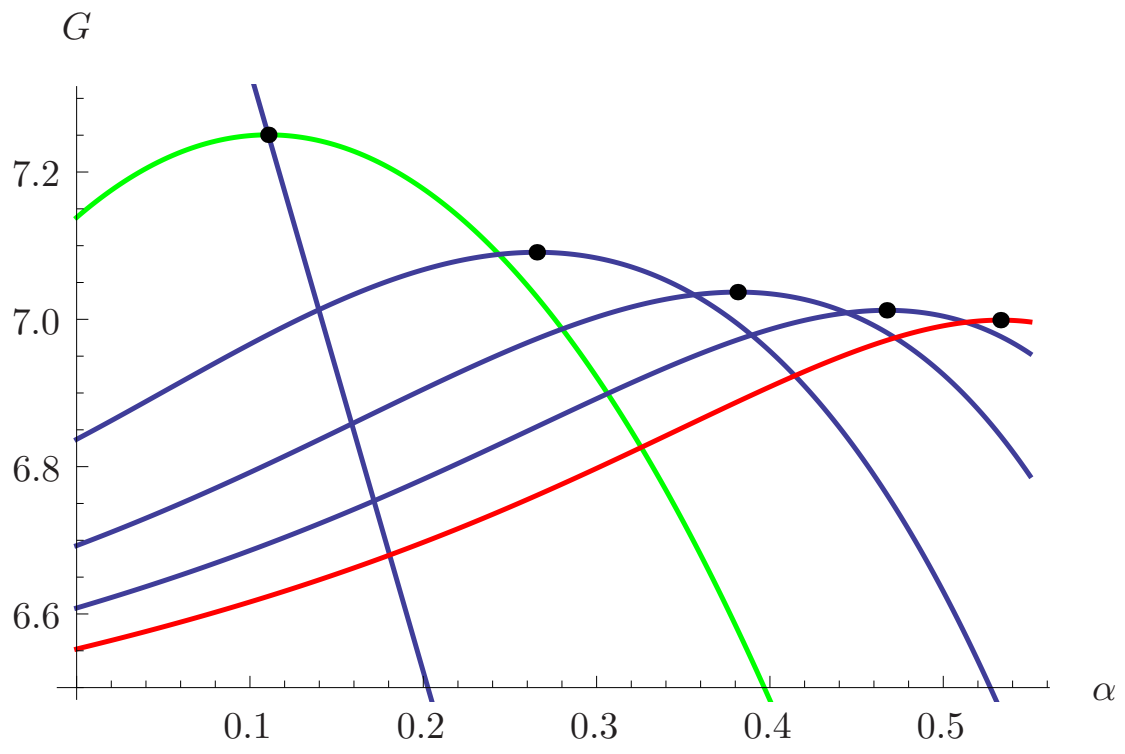

Figure 5. $G$ evaluated at $\theta=10 / 6,10 / 5,10 / 4,10 / 3,10 / 2,10$ (with $\left.y_{0}=1, r=1 / 10, \rho=1 / 20, T=10\right)$.

Quasi-concavity in conjunction with differentiability of $G(\theta, \cdot)$ allows calculating the optimal harvesting share $\alpha^{*}$ using the first order condition

$$
\alpha^{*}: \quad \frac{\partial G(\theta, \alpha)}{\partial \alpha}=0 .
$$

As the explicit expression of this derivative provides little insight, we relegate it to Appendix B, see Equation (B.1). Note however, that due to the lack of quasiconcavity, the optimal time for one round cannot be found by differentiation. Yet, 
as the objective function is convex on any compact interval $[a, b]$ whenever $a$ and $b$ are two adjacent kinks, $G(\cdot, \alpha)$ attains its maximum (if it exists) at either $a$ or $b$ (or both). It thus suffices to compare the values of $G(\cdot, \alpha)$ at a countable number of kinks. Since these kinks accrue whenever the agent adjusts the speed of harvesting so as to manage to complete exactly an integer number of rounds, i.e., the agent's final position $s(T)$ equals $2 \pi$, we have to compare the discounted payoffs from completing exactly $n=1,2, \ldots$ circles within time $T$. The optimal time of circling $\theta^{*}$ must then equal $T / n$ for some suitable $n \in \mathbb{N}$. In the example used in Figures 4 and 5 we set $T=10$, so that the kinks appear at $\theta=10,5,10 / 3,5 / 2,2,10 / 6, \ldots$, that is, at $n=1,2,3,4,5,6, \ldots$, respectively. These $\theta$-values are indicated by dashed vertical lines in Figure 4.

We now exploit the important finding that in an optimal solution the agent travels an integer number of circles $n \in \mathbb{N}$. It follows that $\theta=T / n$ and $\tau=0$. Observe that with $n=T / \theta \in \mathbb{N}$ the objective function $G$, defined by Equation (8), reduces to the simpler form (7). Similarly we may use this observation to simplify the first order condition for the optimal $\alpha$. Provided that $e^{\rho \theta}-\alpha e^{r \theta} \neq 0$, the condition (B.1) reduces to

$$
\begin{array}{r}
\alpha^{n-1} e^{n r \theta}\left(\alpha(n(\alpha-1)+1) e^{r \theta}-(n(\alpha-1)+\alpha) e^{\rho \theta}\right)+e^{(n+1) \rho \theta}-e^{\theta(n \rho+r)}=0 \\
\Leftrightarrow \quad \alpha^{n-1} e^{n \sigma \theta}\left(\alpha((\alpha-1) n+1) e^{\sigma \theta}-\alpha(n+1)+n\right)-\left(e^{\sigma \theta}-1\right)=0
\end{array}
$$

where we have introduced the shorthand $\sigma \equiv r-\rho$. For each given number of rounds of circling $n$, the admissible optimal values of $\alpha$ are given by the positive roots within the unit interval of the first-order condition (9). Note that the optimal value of $\alpha$ depends only on the difference $\sigma$, and not on the individual values of $r$ and $\rho$.

For $n=1$, Equation (9) has no solution, which suggests that the optimal value of $\alpha$ is either zero or unity. As $E(1)$ is linearly decreasing in $\alpha$, the agent chooses $\alpha(1)=0$ and harvests the complete stock of the resource. We investigate this case in detail in the next section.

For $n=2$, the unique admissible solution of Equation (9) is given by

$$
\alpha(2)=\frac{1}{2}\left(1-e^{-\theta \sigma}\right)
$$

from which we infer that $\frac{\partial \alpha(2)}{\partial \sigma}=\frac{1}{2} \theta e^{-\sigma \theta}>0$. For $\sigma=1 / 20$ and $T=10$, and thus $\theta=5$, this formula yields $\alpha(2)=\frac{1}{2}\left(1-e^{-1 / 4}\right) \approx 0.1106$, which brings about the maximum of the green graph of $G$ in Figure 5 and the left maximum of the green graph of $G$ in Figure 4. Note that the maximum is not unique, as for this value of $\alpha$ a single round of circling, i.e., $\theta=10$ brings about the same value of $G$ as $G(5,0.1106)=G(10,0.1106)=7.25046$. However, neither of these constitutes 
a solution of the problem as the total exploitation of the resource with a single round of circling yields $G(10,0)=8.15207$.

For $n=3$ we obtain two roots of the first-order condition,

$$
\alpha_{1,2}(3)=\frac{1}{3} e^{-\sigma \theta}\left(e^{\sigma \theta}-1 \pm \sqrt{e^{\sigma \theta}+e^{2 \sigma \theta}-2}\right) .
$$

Clearly, since $e^{\sigma \theta}+e^{2 \sigma \theta}-2=\left(e^{\sigma \theta}-1\right)^{2}+3\left(e^{\sigma \theta}-1\right)>\left(e^{\sigma \theta}-1\right)^{2}$, only the "plus-root" $\alpha_{1}$ is positive, and thus represents the unique admissible solution. Differentiating $\alpha_{1}(3)$ with respect to $\sigma$ yields

$$
\frac{\partial \alpha_{1}(3)}{\partial \sigma}=\frac{\theta}{\mathcal{N}}\left(4-e^{\sigma \theta}+2 \sqrt{e^{\sigma \theta}+e^{2 \sigma \theta}-2}\right)>\frac{\theta}{\mathcal{N}}\left(2+e^{\sigma \theta}\right)>0,
$$

with $\mathcal{N} \equiv 6 e^{\sigma \theta} \sqrt{e^{\sigma \theta}+e^{2 \sigma \theta}-2}>0$.

Finally, for $n=4$, we obtain three roots of the first-order condition

$$
4 \alpha^{3} e^{3 \sigma \theta}+\alpha^{2}\left(3 e^{2 \sigma \theta}-3 e^{3 \sigma \theta}\right)+\alpha\left(2 e^{\sigma \theta}-2 e^{2 \sigma \theta}\right)-e^{\sigma \theta}+1=0,
$$

and again those within the unit interval represent the admissible solutions.

The analysis of the cases $n=2,3$, and 4 suggests the following results:

- The optimal value of $\alpha$ is increasing in $n$ : The more often the agent chooses to extract from the resource, the lower is the rate of extraction.

- The optimal value of $\alpha$ is increasing in $\sigma$ : The higher the growth rate of the resource (or the lower the agent's discount rate) the lower is the rate of extraction.

The contours of the objective function $G$ are displayed in Figure 6. We have drawn horizontal lines for $\theta=1,5 / 3,2,5 / 2,10 / 3,5$, and 10 , i.e., for $n=$ $10,6,5,4,3,2$, and 1 , respectively. In Figure 6 it is easy to identify the kinks which we have observed in Figure 4. We readily infer from Figure 6 that $G$ has a unique maximum at $(\theta, \alpha)=(10,0)$ yielding $G(10,0)=8.15207$. The agent thus chooses to fully exploit the resource during the single round of circling. Plots corresponding to Figure 6 for $r=1 / 5, r=1 / 2$, and $r=1$, are displayed in Figures 7 , 8 , and 9 respectively. Comparing the results for different values of $r$ shows that a change in the growth rate of the resource does not affect the qualitative features of our results.

It is noteworthy that the structure of the solution of the agent's optimization problem turns out to be robust to the introduction of our spatial framework: with the instantaneous profit (payoff) being linear in the harvest, and the growth of the resource being linear in both the stock and the harvest, the resulting Hamiltonian is linear in $y$ and $h$. This linear structure generically leads to bang-bang solutions if the control $h$ is bounded, and to impulse controls if it is not, with the 


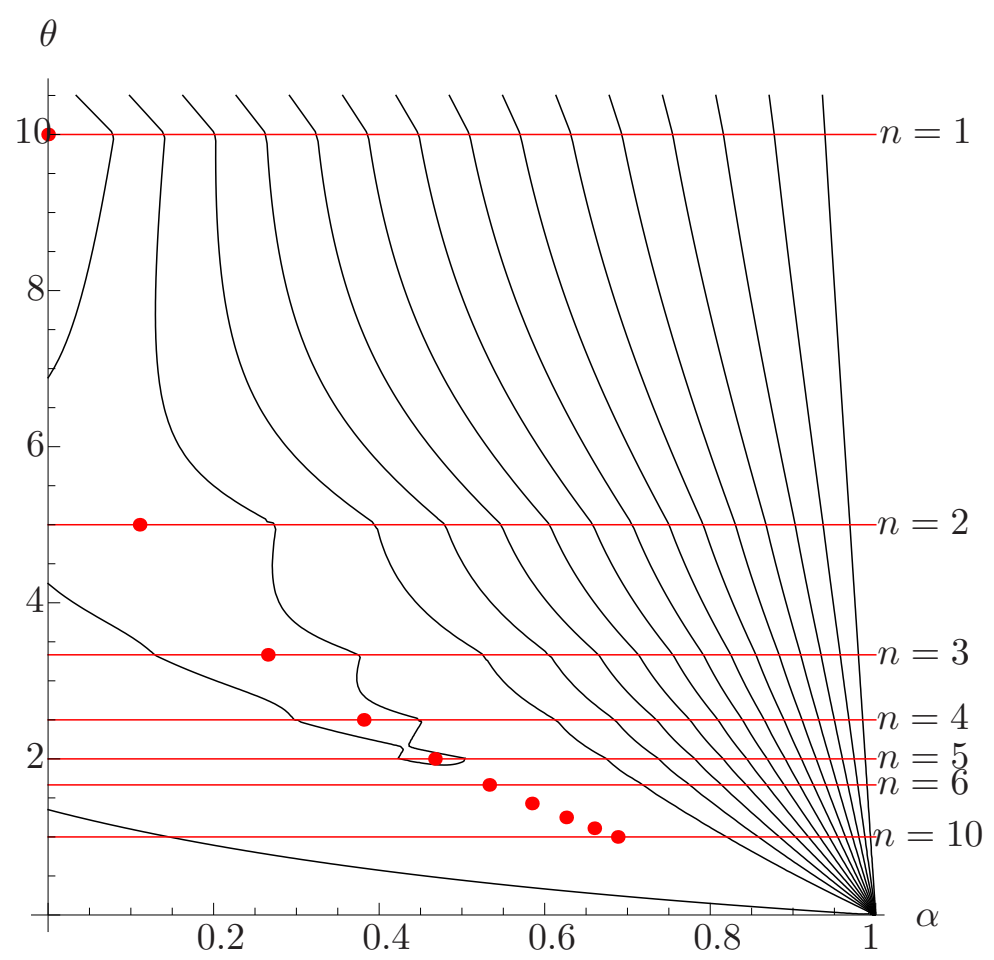

Figure 6. Objective function $G$ with $r=1 / 10\left(y_{0}=1, \rho=\right.$ $1 / 20, T=10)$.

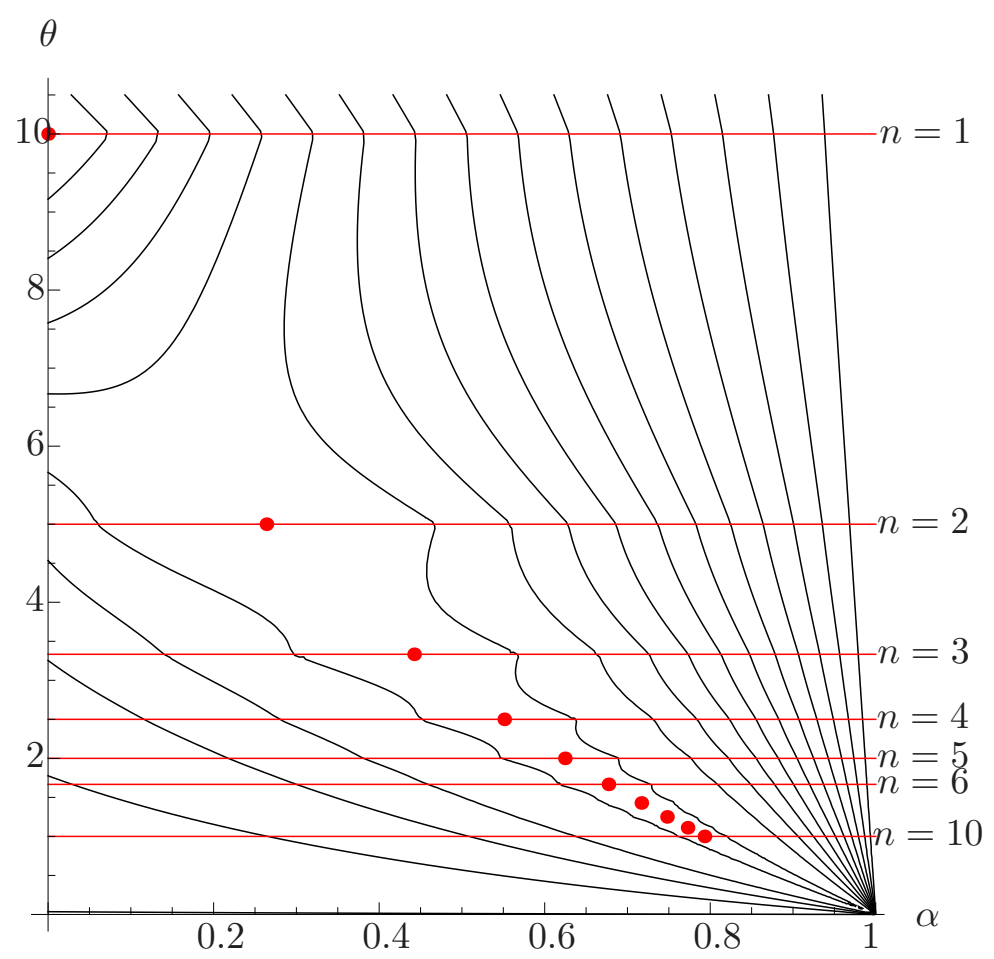

Figure 7. Objective function $G$ with $r=1 / 5\left(y_{0}=1, \rho=\right.$ $1 / 20, T=10)$. 


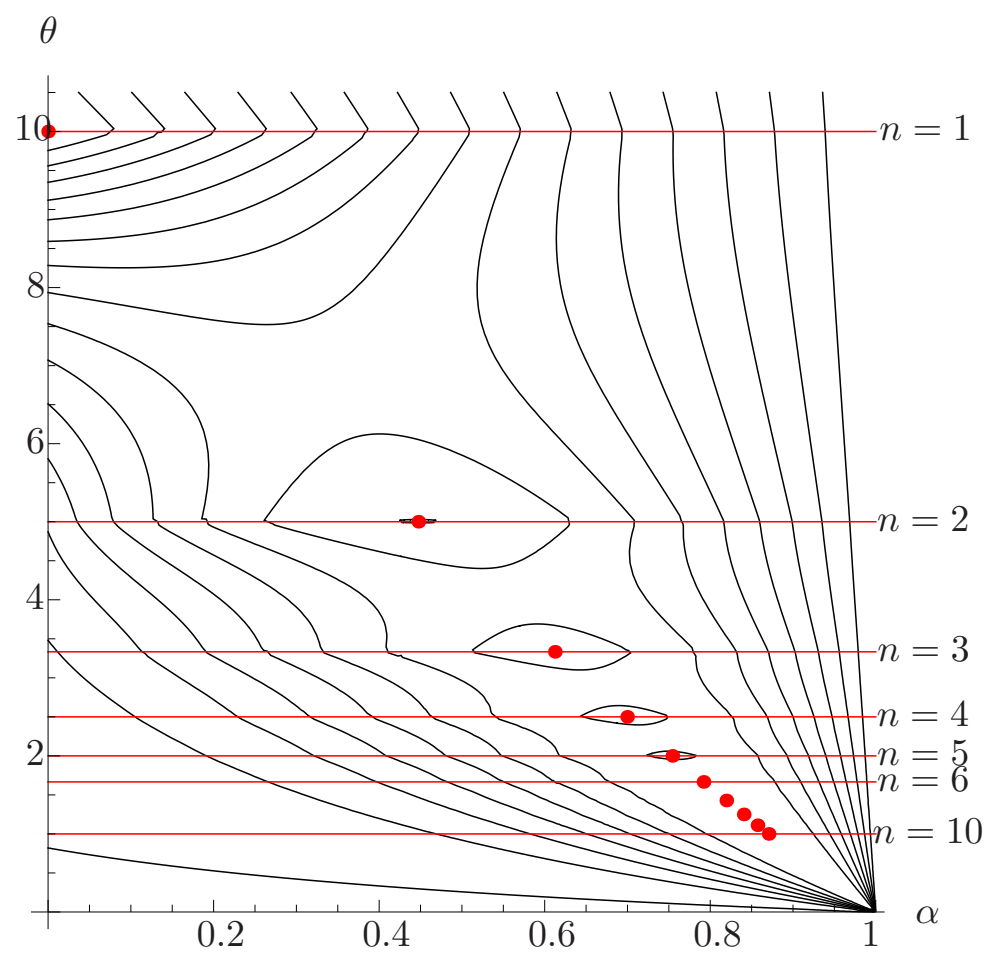

FIgURE 8. Objective function $G$ with $r=1 / 2\left(y_{0}=1, \rho=\right.$ $1 / 20, T=10)$.

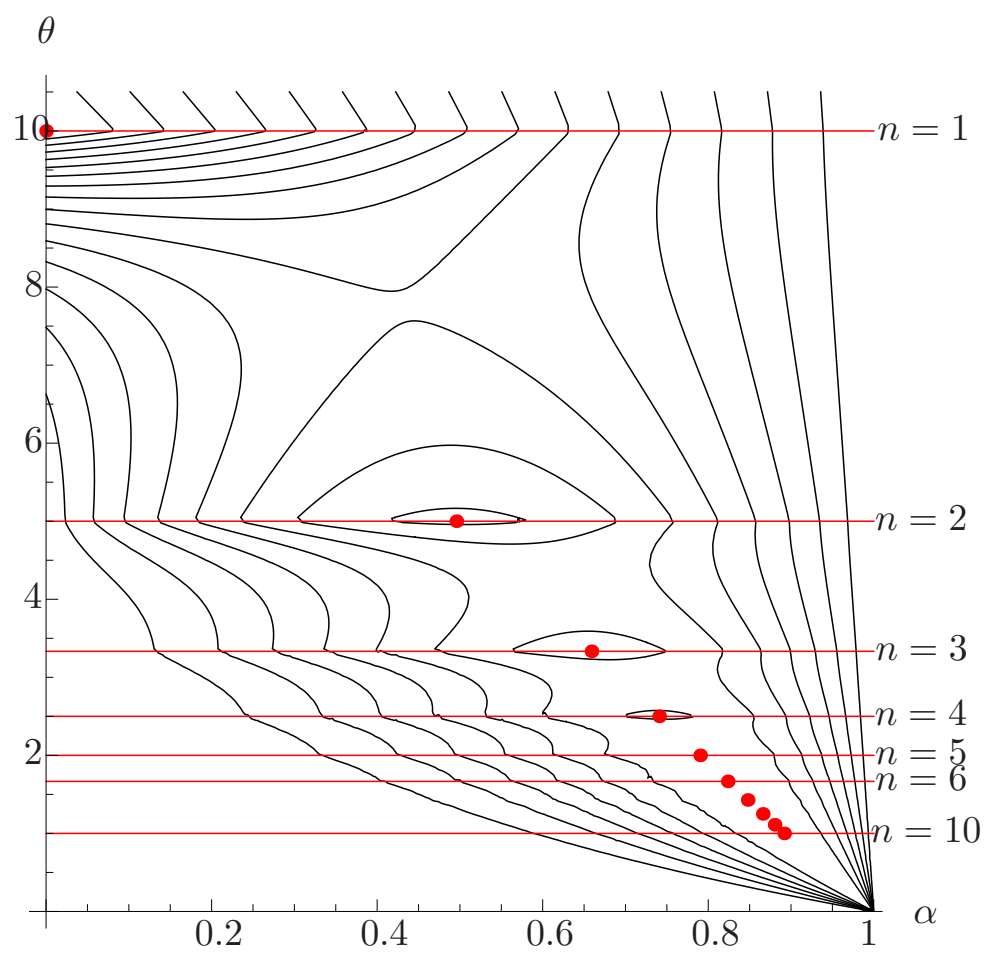

Figure 9. Objective function $G$ with $r=1\left(y_{0}=1, \rho=1 / 20, T=10\right)$. 
optimal harvesting policy depending on whether the net growth rate $\sigma$ is positive or negative. ${ }^{12}$

\section{The Cost of Movement}

Until now we have assumed that the agent can travel on the periphery of the circle at any speed or frequency without incurring a cost. The assumption of a costless choice of speed is innocuous if the cost of movement depends on travelling time only, and not on speed. However, as travelling cost typically depends on the speed chosen, it is necessary to scrutinize whether and how our results change once we take into account such costs. To this end, let us assume that the agent incurs some constant cost $k>0$ per unit of distance. With this specification, travelling costs increase linearly with speed $v$. For example, the travelling cost for $n$ rounds of circling amounts to $n 2 \pi k=v T k$. With this specification, the discounted cost of movement of the first round up to location $x$ equals

$$
\int_{0}^{x} k e^{-\rho t_{1}(\xi)} d \xi=\int_{0}^{x} k e^{-\frac{\rho \theta \xi}{2 \pi}} d \xi=\frac{2 \pi k\left(1-e^{-\frac{\rho x \theta}{2 \pi}}\right)}{\rho \theta} .
$$

Similarly, the discounted cost of movement of the $n$th round up to location $x$ amounts to

$$
\int_{0}^{x} k e^{-\rho t_{n}(\xi)} d \xi=\int_{0}^{x} k e^{-\frac{\rho \theta(2 \pi(n-1)+\xi)}{2 \pi}} d \xi=\frac{2 \pi k e^{-(n-1) \rho \theta}\left(1-e^{-\frac{\rho x \theta}{2 \pi}}\right)}{\rho \theta} .
$$

Summing the $Q(T, \theta)=\left\lfloor\frac{T}{\theta}\right\rfloor$ complete rounds plus the residual part $\bmod (T, \theta)$ of the last round we obtain aggregate discounted cost of movement as

$$
\frac{2 \pi k\left(1-e^{-n \rho \theta}\right)}{\rho \theta}=\frac{2 \pi k\left(1-e^{-\rho T}\right)}{\rho \theta}=: C(\theta) .
$$

Note that $C$ smoothly depends on the speed of movement $\theta$. Unlike the aggregate discounted revenue $G(\cdot, \alpha)$ given by Equation (8), $C$ does not exhibit kinks and is differentiable everywhere. For that reason the previously observed kinks of $G(\cdot, \alpha)$ carry over into kinks of the profit function $G(\cdot, \alpha)-C(\cdot)$ for any given value of $\alpha \in(0,1)$.

As $C$ does not depend on $\alpha$, the conditionally optimal harvesting or conservation rate $\alpha(n)$ is not affected by the introduction of a cost of movement. Finally, because $C^{\prime}<0$, the discounted profit decreases with lower values of $\theta$. As a consequence, the introduction of a cost of movement strengthens the agent's incentives to choose a high value of $\theta$. To sum up: the introduction of a speed-dependent cost of motion strengthens our previous result that the agent chooses a complete

\footnotetext{
${ }^{12}$ For more details on this, see, for example, Feichtinger and Hartl (1986), Conrad and Clark (1987), Clark (1990) or Kamien and Schwartz (1992).
} 
number of rounds $n=T / \theta$ and that without any additional constraint on $n$ or $\theta$ the agent's profit is maximized by the choices of $n=1$ and $\alpha(1)=0$. The agent visits each location of the resource only once and the stock is completely depleted following this first visit.

Next we wish to explore the consequences of introducing harvest dependent costs. Assume that the speed of circling can be freely chosen at no cost, but that the harvesting cost amounts to $C(\alpha)$ with $C^{\prime}<0$ and $C(1)=0$. This reflects the idea that a higher harvesting rate increases the cost of harvesting while full conservation of the resource can be accomplished at no cost. As harvesting costs are independent of $\theta$, it is easiest to interpret $C(\alpha)$ as a cost per unit of time and to integrate the harvesting cost over time:

$$
\int_{0}^{T} c(\alpha) e^{-\rho t} d t=\frac{1-e^{-\rho T}}{\rho} C(\alpha)
$$

The discounted marginal harvesting cost amounts to $\frac{1-e^{-\rho T}}{\rho} c^{\prime}(\alpha)$, which has to be subtracted from the marginal yield of harvesting derived above (see Equation (B.1)). Obviously for a marginal cost $c^{\prime}$ that becomes sufficiently large as $\alpha$ approaches zero, the complete depletion result obtained above will cease to hold and interior solutions (i.e., $\alpha>0$ ) will result. Moreover a suitable specification of $c$ may even lead to a reversal of the negative relation between the optimal value of $\alpha$ and $\theta$. As we see no reason for the cost function to exhibit these features, we will not further discuss what we believe are rather pathological cases.

\section{Detailed Analysis of the First Period-With a Capacity Constraint}

Assume that the agent chooses to circulate at most once, arriving at each point $x \in[0,2 \pi]$ either once or never. We presume here that the agent chooses at most the minimal speed required to travel the full periphery $2 \pi$ within the given time $T$. Let $\phi \in[0,2 \pi]$ denote the location of the agent at time $T$, i.e., $\phi=s(T)$. To be more precise, we should write $\phi(v, T)$ instead of $\phi$, since the final position of the agent depends on both the chosen speed and the travelling time. Using Equation (6), the total harvest amounts to

$$
E(1, \phi)=\int_{0}^{\phi}(1-\alpha) y_{0} \exp \left(\sigma \theta \frac{x}{2 \pi}\right) d x=(1-\alpha) \frac{2 \pi y_{0}}{\sigma \theta}\left(\exp \left(\sigma \theta \frac{\phi}{2 \pi}\right)-1\right) .
$$

Replacing $\theta$ by $v$ we may express the total harvest as

$$
E(1, \phi)=(1-\alpha) y_{0} \frac{v}{\sigma}\left(\exp \left(\frac{\sigma \phi}{v}\right)-1\right)=(1-\alpha) y_{0} \frac{v}{\sigma}\left(e^{\sigma T}-1\right) .
$$

The last equality follows from the fact that with constant speed $v$, the agent arrives at location $\phi$ after travelling time $T$, i.e., $\phi=v T$. 
5.1. Non-Binding capacity constraint. Suppose that the agent's harvesting capacity $\bar{h}$ is sufficiently high so that the capacity constraint is never binding within the time interval $[0, T]$, i.e., $\bar{h} \geq y_{0} e^{r \theta} \geq y_{0} e^{r t_{1}(\phi)}$. Clearly with a nonbinding harvesting capacity constraint, the agent depletes the total stock at each $x \in[0, \phi]$, for the agent will never come back to benefit from any future growth of the resource. Hence, with $\alpha=0$, the total discounted harvest equals

$$
\left.E(1, \phi)\right|_{\alpha=0}=y_{0} \frac{v}{\sigma}\left(\exp \left(\frac{\sigma \phi}{v}\right)-1\right)=y_{0} \frac{v}{\sigma}\left(e^{\sigma T}-1\right) .
$$

If we disregard the cost of movement, the problem is nondescript as the agent would choose the maximally admissible speed, i.e., the minimum speed required to complete a full circle that is, choose $v^{*}=\bar{v} \equiv 2 \pi / T$, and the final location of the agent at time $T$ would be $\phi\left(v^{*}\right)=2 \pi$. Correspondingly, the optimal discounted harvest equals

$$
G^{*}=\left.E(1,2 \pi)\right|_{\alpha=0}=\left.E(1)\right|_{\alpha=0}=y_{0} \frac{2 \pi}{\sigma T}\left(e^{\sigma T}-1\right) .
$$

However, with the cost of movement being some increasing and strictly convex function of speed $c:[0, \bar{v}] \rightarrow \mathbb{R}_{+}$, the agent has to solve

$$
\max _{v} y_{0} \frac{v}{\sigma}\left(e^{\sigma T}-1\right)-c(v)
$$

yielding the optimal speed

$$
v^{*}=c^{\prime-1}\left(\frac{y_{0}}{\sigma}\left(e^{\sigma T}-1\right)\right)
$$

if and only if

$$
c^{\prime}(0)<\frac{y_{0}}{\sigma}\left(e^{\sigma T}-1\right)<c^{\prime}(\bar{v})
$$

5.2. Binding capacity constraint. In order to broaden the applicability and enhance the realism of our model, we shall now assume that the agent's harvesting capacity $\bar{h}$ is sufficiently low so that the capacity constraint is always binding, i.e., $\bar{h} \leq y_{0}$, and the agent harvests at full capacity for all $t \in \mathcal{T}$. Hence, the total discounted harvest equals

$$
\int_{0}^{\phi} \exp \left(-\rho \frac{\theta x}{2 \pi}\right) \bar{h} d x=\frac{2 \pi \bar{h}}{\rho \theta}\left(1-e^{-\frac{\rho x \theta}{2 \pi}}\right)=\frac{\bar{h} v}{\rho}\left(1-e^{-\rho T}\right) .
$$

Disregarding the cost of movement, the problem is uninteresting as the agent would choose $v^{*}=\bar{v} \equiv 2 \pi / T$. With some convex cost function $c$, though, the agent has to solve

$$
\max _{v} \frac{\bar{h} v}{\rho}\left(1-e^{-\rho T}\right)-c(v)
$$

yielding the optimal speed

$$
v^{*}=c^{-1}\left(\frac{\bar{h}}{\rho}\left(1-e^{-\rho T}\right)\right)
$$


5.3. Partially binding capacity constraint. Let us now turn to the more interesting case that the agent's harvesting capacity $\bar{h}$ is intermediate, i.e., $y_{0}<$ $\bar{h}<y_{0} e^{r \theta}$. In this case the solution is a straightforward combination of the two polar cases above. Define the critical level at which the stock of the resource equals the agent's harvesting capacity $\hat{x}(\bar{h})$ as the solution of

$$
y_{0} \exp \left(r \frac{\theta x}{2 \pi}\right)=\bar{h}
$$

Thus, $\hat{x}(\bar{h})$, or for short $\hat{x}$, equals

$$
\hat{x}(\bar{h})=\frac{2 \pi}{r \theta} \log \left(\frac{\bar{h}}{y_{0}}\right) .
$$

Up to time $t_{1}(\hat{x}(\bar{h}))$, the agent is able to harvest the total stock of the resource. For the remaining time, i.e., given by the time interval $\left[t_{1}(\hat{x}(\bar{h})), T\right]$, the agent is capacity constrained. The total discounted harvest is then given by

$$
\begin{aligned}
G(\phi) & =\int_{0}^{\hat{x}} y_{0} \exp \left(\sigma \frac{\theta x}{2 \pi}\right) d x+\int_{\hat{x}}^{\phi} \bar{h} \exp \left(-\rho \frac{\theta x}{2 \pi}\right) d x \\
& =y_{0} \frac{v}{\sigma}\left(\frac{r}{\rho}\left(\frac{\bar{h}}{y_{0}}\right)^{\frac{\sigma}{r}}-\frac{\sigma}{\rho} \frac{\bar{h}}{y_{0}} e^{-\rho T}-1\right),
\end{aligned}
$$

where we used $\phi=v T$ and $\theta=2 \pi / v$. Since $G$ is linear in $v$, the optimal speed is either 0 or $2 \pi / T$, depending on whether the term in brackets on the right-hand side of Equation (13) is negative or positive. Since we know that $G$ is positive, the term in brackets must be positive as well - and the agent therefore chooses the maximal speed, that is, the speed which allows completing one full round, i.e., $v^{*}=2 \pi / T$.

\section{Variable Harvesting Rates}

We next relax the assumption of a constant harvesting rate and allow the agent to choose any conservation rate (negative harvesting rate) $\alpha(t) \in[0,1]$. We show that even in this unconstrained case, the agent will not choose an interior solution, but the optimal conservation rate will be either 0 or 1 (or is indeterminate as the stock is already totally depleted). In order to demonstrate this result, it is convenient to consider some arbitrary but fixed location $x \in \mathcal{S}$, and to let the agent maximize the aggregate discounted harvesting revenue collected at location $x$ over the planning period $\mathcal{T}$.

Since $x$ is fixed, we suppress it as an argument. Accordingly, the arrival time at location $x$ in the $n$th harvesting period is denoted by $t_{n}$, and the respective 
conservation rate, by $\alpha_{n} \equiv \alpha\left(t_{n}\right)$. The present value of the harvesting revenue from the $n$th arrival at location $x$ then equals

$$
y_{0}\left(1-\alpha_{n}\right) e^{t_{n}(r-\rho)} \prod_{i=1}^{n-1} \alpha_{i} .
$$

Summing over $N$ periods of harvesting and using the vector notation $\boldsymbol{\alpha} \equiv$ $\left(\alpha_{1}, \ldots, \alpha_{N}\right)$, the aggregate discounted harvest amounts to

$$
G(\boldsymbol{\alpha})=\sum_{n=1}^{N} y_{0}\left(1-\alpha_{n}\right) e^{t_{n}(r-\rho)} \prod_{i=1}^{n-1} \alpha_{i} .
$$

Since this objective function $G$ is a multilinear mapping, ${ }^{13}$ it follows that the partial derivative of $G$ with respect to $\alpha_{n}$ is independent of $\alpha_{n}$. For this reason, the optimal value of $\alpha_{n}$ is either 0 or 1 , or it is indeterminate.

We next show that the optimal conservation (negative harvesting) profile is given by $\boldsymbol{\alpha}^{+}=(1, \ldots, 1,0)$, if $\sigma \equiv r-\rho>0$; and by any $\boldsymbol{\alpha}^{-}$of the type $(0, \bullet, \ldots, \bullet)$, if $\sigma<0$. (A bullet $\bullet$ at position $i$ means that $\alpha_{i}$ is indeterminate and can thus take any value in $[0,1]$.)

Evaluating the gradient of $G$ at the proposed maximizer $\boldsymbol{\alpha}^{+}$yields

$$
\nabla G\left(\boldsymbol{\alpha}^{+}\right)=y_{0}\left(e^{t_{N} \sigma}-e^{t_{1} \sigma}, \ldots, e^{t_{N} \sigma}-e^{t_{N-1} \sigma},-e^{t_{N} \sigma}\right)
$$

Accordingly, we have

$$
\operatorname{sgn} \nabla G\left(\boldsymbol{\alpha}^{+}\right)= \begin{cases}(1, \ldots, 1,-1), & \text { if } \sigma>0 \\ (-1, \ldots,-1), & \text { if } \sigma<0\end{cases}
$$

implying that the corner solution $\boldsymbol{\alpha}^{+}$is optimal for $\sigma>0$ and that $\mathbf{0} \equiv(0, \ldots, 0)$ is optimal for $\sigma<0$. In the latter case, any vector $\boldsymbol{\alpha}^{-}$of the type $(0, \bullet, \ldots, \bullet)$ is optimal, as $G(\mathbf{0})=G(0, \bullet, \ldots, \bullet)=y_{0} e^{t_{1} \sigma}$ for any choice of $\alpha_{2}, \ldots, \alpha_{N}$.

Alternatively, it is easy to check that

$$
G(\underbrace{1, \ldots, 1}_{n-1}, 0, \underbrace{\bullet, \ldots, \bullet}_{N-n})=y_{0} e^{t_{n} \sigma} .
$$

Since $t_{1}<t_{2}<\ldots<t_{N}$, we have $y_{0} e^{t_{n} \sigma}$ is maximal for $n=N$ if $\sigma>0$, and it is maximal for $n=1$ if $\sigma<0$.

We have thus shown that with $\sigma>0$, the agent will let the resource grow freely so as to be able to totally deplete it in the last round, i.e., the agent will not harvest the resource in the first $N-1$ rounds at all. "Waiting" until the last round by circling without harvesting is optimal as the growth rate of the stock exceeds the agent's discount rate. Here the agent is sufficiently patient so that the

\footnotetext{
${ }^{13}$ That is, for each $n=1, \ldots, N$ the function $G_{n}\left(\alpha_{-n}\right):[0,1] \rightarrow \mathbb{R}_{+}: \alpha_{n} \mapsto G_{n}\left(\alpha_{n}, \alpha_{-n}\right)$ is linear.
} 
total discounted revenue is maximized by letting the resource grow for as long as possible. If $\sigma<0$, i.e.. the agent is relatively impatient, the reverse argument holds.

Finally, whenever there is only one round of travelling - either because the agent chooses to go for only one round or because the agent is exogenously restricted to visiting each location only once - the first and the last round coincide. In this case, solutions $\boldsymbol{\alpha}^{+}$and $\boldsymbol{\alpha}^{-}$coincide, implying that there is only one fully exploitative round, $\boldsymbol{\alpha}^{+}=(0)=\boldsymbol{\alpha}^{-}$, irrespective of the sign of $\sigma$. In this way, the analysis of this section confirms our result of Section 3.2, where we found that the constant conservation rate is given by $\alpha(1)=0$, i.e., if there is only one round of harvesting.

\section{Conclusion}

As argued in the Introduction, we believe that our paper makes an important contribution to the technical side of renewable resource harvesting models that simultaneously look at a time and a spatial dimension, and also has policy implications. Countering the startling previous lack of attention by economists and given the prominence of spatial dynamic systems in the hard sciences, as observed by Wilen (2007), we have provided an attempt at a dynamic model with continuous variables and have solved it for the steady state. To this end, we applied the frequently used example of a fishery with spatially distributed fish.

On the technical side, we presented a simple way to introduce continuous spatial dynamics into models of renewable resource harvesting in continuous time. Models of optimal harvesting in fisheries economics are often specified in discrete time in order to take into account the fact that "both reproductive and fishing activities may have clear seasonal characteristics" (Tahvonen, 2009, p. 282). Despite its setting in continuous time, in our model the attractive seasonal characteristic prevails due to its cyclical structure.

On the policy side, we are able to show that left to their own devices, agents will choose to go for one, fully exploitative round of harvesting therefore leading to the immediate, i.e., at the time of the agent's first arrival, extinction of the species. One way of preventing this socially harmful result is to constantly monitor and control the actual catch of the agent. Alternatively, a bound on exploitative behaviour can be implemented by directly restricting the total catch by a quota. Quotas as such however are relatively crude instruments and do not take into account the growth potential of young fish and thus the reproduction capacity of a species. Also, it is very hard to monitor the actual by-catch that is disposed of at sea, which is a well-known problem. Our model sheds light on the more 
flexible policy alternative of a minimum mesh size regulation, provided that the stock consists of a heterogeneous population. ${ }^{14}$ This policy takes into account the actual heterogeneity of populations and their idiosyncratic growth potentials and thus has numerous advantages over quotas (see Jones, 1984, for details). The socially detrimental maximizing behaviour of the agent can only be pursued if fishing with the minimal mesh size is feasible. If the minimal mesh size is bounded below, incentives change and the agent will become concerned about the growth potential of the stock that is left behind. Thus the social objective of preserving the species for the future can be served.

We are convinced that the simplicity of our model and its implications warrant the interest of the economics discipline beyond the realm of resource and environmental economics. Our model has a certain proximity to the two seminal papers of Hotelling $(1929,1931)$. The wealth of applications of his static model (1929) equally applies to our truly dynamic context. Hence, an extension of the present dynamic model to an oligopoly situation with non-competitive behaviour is immediate. Moreover, introducing further realistic properties such as potential movement (or diffusion) of the resource or acceleration of the agent (boat) may enhance the insights from spatial resource models.

As shown by Belyakov, Davydov, and Veliov (2013), a similar spatial setup with a different growth process and different long run objectives can lead to situations in which the renewable resource is completely depleted in some areas but not in others. Hence, the feasibility of a sustainability doctrine that is based on the persistent existence of the renewable resource in all its original locations is put into question and important issues about adequate alternative definitions are raised. Such "exhausted areas" could realistically be expected to be repopulated if the resource itself can move, as is modelled in the works of Brock and Xepapadeas (2010) and Brock, Xepapadeas, and Yannacopoulos (2013). Such a diffusion of the resource clearly enhances the insights from spatial resource models. Similarly, we also expect that recent developments in age-structured population models (see Hritonenko and Yatsenko, 2006; and Anita, 1998, for references) may fruitfully contribute to a dynamic analysis of the economics of spatially distributed renewable resources. Finally, varying the harvesting capacity by allowing varied investment behaviour on the part of the agent, e.g. investments of the fisher in boats and fishing nets without and with uncertainty, as investigated by Brennan and Schwartz (1985), constitute challenging paths for future research.

\footnotetext{
${ }^{14}$ In our model, the stock of the resource may be interpreted as the aggregate of a heterogeneous population featuring a constant composition. Adding real heterogeneity into our spatial model, though, will lead to a substantial increase in the complexity of the analysis, and is thus beyond the scope of the present paper.
} 


\section{References}

S. Anita (1998), Optimal Harvesting for a Nonlinear Age-dependent Population Dynamics, Journal of Mathematical Analysis and Applications, 226, 6-22.

A. O. Belyakov, A. Davydov, and V. M. Veliov (2013), Optimal Cyclical Exploitation of Renewable Resources, ORCOS Research Report 2013-07, Vienna University of Technology.

M. J. Brennan and E. Schwartz (1985), Evaluating Natural Resource Investments, Journal of Business, 58, 135-157.

W. Brock and A. Xepapadeas (2010), Pattern Formation, Spatial Externalities and Regulation in Coupled Economic-ecological Systems, Journal of Economics and Management, 59, 149-164.

W. Brock, A. Xepapadeas, and A. Yannacopoulos (2013), Robust Control of a Spatially Distributed Commercial Fishery, Working Paper, Athens University of Economics and Business.

C. W. Clark (1990), Mathematical Bioeconomics, second ed., Wiley, New York.

J. M. Conrad (2010), Resource Economics, second ed., Cambridge University Press, Cambridge, UK.

J. M. Conrad and C. W. Clark (1987), Natural Resource Economics, Cambridge University Press, Cambridge.

C. Costello and S. Polasky (2008), Optimal Harvesting of Stochastic Spatial Resources, Journal of Environmental Economics and Management, 56, 1-18.

R. T. Deacon, D. S. Brookshire, A. C. Fisher, A. V. Kneese, C. D. Kolstad, D. Scrogin, V. K. Smith, M. Ward, and J. Wilen (1998), Research Trends and Opportunities in Environmental and Natural Resource Economics, Environmental and Resource Economics, 11, 383-397.

G. Feichtinger and R. Hartl (1986), Optimale Kontrolle Ökonomischer Prozesse, Walter de Gruyter, Berlin.

H. S. Gordon (1954), Economic Theory of a Common Property Resource: The Fishery, Journal of Political Economy, 75, 124-142.

R. Hannesson (2011a), Game Theory and Fisheries, Annual Review of Resource Economics, 3, 118-202.

R. Hannesson (2011b), Rights Based Fishing on the High Seas: Is it Possible?, Marine Policy, 35, 667-674.

H. Hotelling (1929), Stability in Competition, The Economic Journal, 39, 41-57. 
H. Hotelling (1931), The Economics of Exhaustible Resources, Journal of Political Economy, 39, 137-175.

N. Hritonenko and Y. Yatsenko (2006), Optimization of Harvesting Return from Age-Structured Population, Journal of Bioeconomics, 8, 167-179.

R. Jones (1984), Mesh size regulation and its role in fisheries management, in: FAO, (1984) Papers Presented at the Expert Consultation on the Regulation of Fishing Effort (Fishing Mortality), Rome, 17-26 January 1983. A preparatory meeting for the FAO World Conference on fisheries management and development. FAO Fish. Rep., (289) Supp 1.2: Available at:

http://www.fao.org/docrep/005/AC749E/AC749E00.htm (last used 24.10.2012).

M. I. Kamien and N. L. Schwartz (1992), Dynamic Optimization, second ed., Elsevier, North Holland, Amsterdam.

J. B. Kellner, I. Tetreault, S. D. Gaines, and R. M. Nisbet (2007), Fishing the Line near Marine Reserves in Single and Multispecies Fisheries, Ecological Applications, 17(4), 1039-1054.

M. Neubert (2003), Marine Reserves and Optimal Harvesting, Ecology Letters, $6,843-849$.

M. Neubert and G. E. Herrera (2008), Triple Benefits from Spatial Resource Management, Theoretical Ecology, 1, 5-12.

R. Perman, Y. Ma, M. Common, D. Maddison, and J. McGilvary (2011), Natural Resource and Environmental Economics, 4th edition, Pearson.

E. J. Z. Robinson, J. C. Williams, and H. J. Albers (2002), Land Economics, 78(2), 260-271.

E. J. Z. Robinson, H. J. Albers, and J. C. Williams (2008), Spatial and Temporal Modeling of Community Non-Timber Forest Extraction, Journal of Environmental Economics and Management, 56(3), 234-245.

J. N. Sanchirico and J. E. Wilen (2005), Optimal Spatial Management of Renewable Resources: Matching Policy Scope to Ecosystem Scale, Journal of Environmental Economics and Management, 50, 23-46.

A. D. Scott (1955), The Fishery: The Objectives of Sole Ownership, Journal of Political Economy, 63, 116-124.

M. D. Smith, J. N. Sanchirico, and J. E. Wilen (2009), The Economics of Spatial-dynamic Processes: Applications to Renewable Resources, Journal of Environmental Economics and Management, 57, 104-121.

O. Tahvonen (2009), Economics of Harvesting Age-structured Fish Populations, Journal of Environmental Economics and Management, 58, 281-299. 
J. E. Wilen (2007), Economics of Spatial-dynamic Processes, American Journal of Agricultural Economics, 89, 1134-1144. 


\section{Appendix A.}

We know that the total discounted harvest $\sum_{i=1}^{n} E(i)$, given by Equation (7), necessarily has to be positive. Since

$$
(1-\alpha) \frac{2 \pi y_{0}}{(r-\rho) \theta}\left(e^{(r-\rho) \theta}-1\right) \geq 0 \quad \forall r, \rho \in \mathbb{R}_{+},
$$

any parameter restriction on $\sigma:=r-\rho$ must result from the non-negativity of the expression

$$
\psi(n):=\frac{\alpha^{n} e^{n(r-\rho) \theta}-1}{\alpha e^{(r-\rho) \theta}-1} .
$$

Let $\varphi(n):=\alpha^{n} e^{n(r-\rho) \theta}-1$. It then follows from Equation (7) that $\sum_{i=1}^{n} E(i)>$ $0 \Leftrightarrow \psi(n) \equiv \varphi(n) / \varphi(1)>0$, and thus $\psi^{\prime}(n)=\varphi^{\prime}(n) / \varphi(1)$, with $\varphi^{\prime}(n)=$ $\alpha^{n} e^{n \theta(r-\rho)}(\log (\alpha)+\theta(r-\rho))$. Since $\log (\alpha)+\theta(r-\rho)>0 \Leftrightarrow \varphi(1)=\alpha e^{(r-\rho) \theta}-1>0$, we infer that

$$
\psi^{\prime}(n)=\varphi^{\prime}(n) / \varphi(1)>0 \quad \forall r, \rho \in \mathbb{R}_{+} .
$$

Actually, we have that $\psi(0)=0$ and $\psi(1)=1$. We thus conclude that $\psi$ is positive and strictly increasing for all values of $r$ and $\rho$, although we do not know whether $\varphi$ is decreasing (and negative) or increasing (and positive). Hence, the only parameter restriction on $\sigma$ amounts to

$$
\alpha e^{(r-\rho) \theta}-1 \neq 0 \Leftrightarrow e^{\rho \theta}-\alpha e^{r \theta} \neq 0 . \Leftrightarrow \sigma \equiv r-\rho \neq \frac{\log \alpha}{\theta} .
$$

This condition is given on page 14, immediately before Equation (9).

\section{Appendix B.}

Differentiating $G(\theta, \alpha)$ with respect to the second argument yields

$$
\begin{aligned}
& \frac{2 \pi y_{0}}{\theta \sigma\left(e^{\rho \theta}-\alpha e^{r \theta}\right)^{2}}\left(-\alpha^{n} e^{n r \theta-\rho T}\left(-\left(\alpha^{2}-1\right) e^{2 r \theta+\rho \tau}-2 \alpha e^{r(\theta+\tau)+\rho \theta}\right.\right. \\
& \left.+2(\alpha-1) e^{r \theta+\rho(\theta+\tau)}+e^{r \tau+2 \rho \theta}+\alpha^{2} e^{r(2 \theta+\tau)}\right) \\
& +n(\alpha-1) \alpha^{n-1}\left(\alpha e^{r \theta}-e^{\rho \theta}\right) e^{n \theta \sigma-\rho \tau}\left((\alpha-1) e^{r \theta+\rho \tau}+e^{r \tau+\rho \theta}-\alpha e^{r(\theta+\tau)}\right) \\
& \left.-2 e^{\theta(r+\rho)}+e^{2 r \theta}+e^{2 \rho \theta}\right)=0 .
\end{aligned}
$$




\section{Appendix C.}

In order to show that $(n, \alpha)=(1,0)$ is the maximizer of $G$, we have to compare $G(i, \alpha(i))$ for all $i=1,2, \ldots$. We first show that $G(1, \alpha(1))>G(2, \alpha(2))$. (The values $\alpha(1)$ and $\alpha(2)$ are given on page 14.)

$$
\begin{aligned}
G(1, \alpha(1))>G(2, \alpha(2)) \\
\Leftrightarrow \frac{2 \pi y_{0}\left(e^{T \sigma}-1\right)}{T \sigma}>\frac{\pi y_{0} e^{-\frac{T \sigma}{2}}\left(e^{\frac{T \sigma}{2}}-1\right)\left(e^{\frac{T \sigma}{2}}+1\right)^{2}}{T \sigma} \\
\Leftrightarrow \quad \frac{2 e^{\frac{T \sigma}{2}}}{e^{\frac{T \sigma}{2}}+1}>1 .
\end{aligned}
$$

The next step is to show that $G(2, \alpha(2))>G(3, \alpha(3))$, which is equivalent to

$$
\frac{\pi y_{0} e^{-b / 2}\left(e^{b / 2}-1\right)\left(e^{b / 2}+1\right)^{2}}{T \sigma}
$$$$
>\frac{2 \pi y_{0} e^{-b}\left(e^{b / 3}-1\right)}{9 T \sigma}\left(-\sqrt{-2 e^{2 b / 3}+e^{b}+e^{4 b / 3}}+e^{b / 3}+2 e^{2 b / 3}\right)
$$$$
\times\left(e^{b / 3}\left(2 \sqrt{-2 e^{2 b / 3}+e^{b}+e^{4 b / 3}}+5\right)+2 e^{2 b / 3}+2 e^{b}+\sqrt{-2 e^{2 b / 3}+e^{b}+e^{4 b / 3}}\right)
$$$$
\Leftrightarrow-4 e^{b}\left(\sqrt{-2 e^{2 b / 3}+e^{b}+e^{4 b / 3}}+6\right)-9 e^{b / 6}+7 e^{2 b / 3}+9 e^{7 b / 6}-2 e^{4 b / 3}+5 e^{5 b / 3}
$$$$
+e^{b / 3}\left(12 \sqrt{-2 e^{2 b / 3}+e^{b}+e^{4 b / 3}}+14\right)-8 \sqrt{-2 e^{2 b / 3}+e^{b}+e^{4 b / 3}}>0,
$$

where $b \equiv \sigma T$. In a similar manner we may proceed step by step, yet with the terms becoming more and more involved. A numerical analysis readily shows that $G$ is decreasing as the number of rounds increases: more formally, that $\tilde{G}(n) \equiv$ $G(n, \alpha(n))$ is a strictly decreasing function. This is illustrated in Figure 10 for $n=1,2,3$.

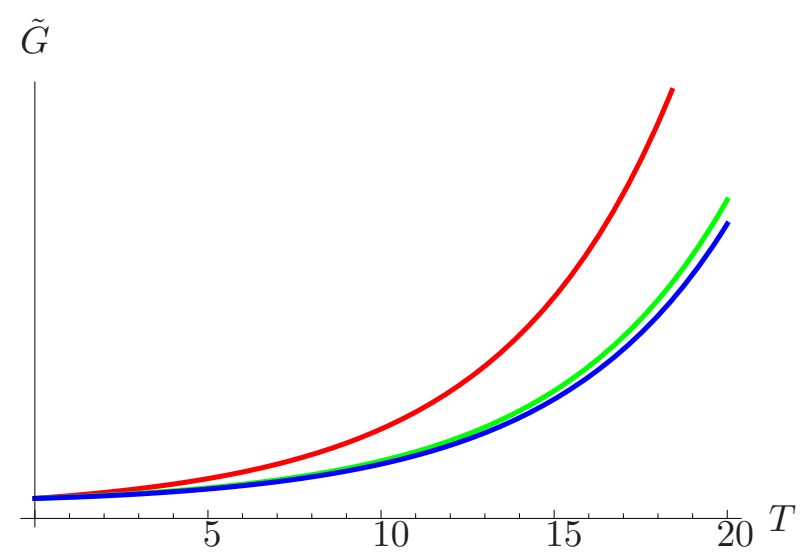

Figure 10. Objective function $\tilde{G}(n) \equiv G(n, \alpha(n))$ for $n=1,2,3$ (red, green, blue). 